\title{
Medición de competencias transversales en una asignatura de grado
}

\author{
Jordi Capó Vicedo ${ }^{a}$, Antonio Giménez-Morera ${ }^{\mathrm{b}}$, Amparo Bonet ${ }^{\mathrm{c}}$
}

${ }^{a}$ Centre for Research in Business Management (CEGEA), Universitat Politècnica de València, Spain. jorcavi0@esp.upv.es, b Universitat Politècnica de València, Department of Economy and Social Sciences, Camino de Vera s/n, 46022, Valencia, Spain angimo1@doctor.upv.es, c Universitat Politècnica de València, Department of Economy and Social Sciences, Camino de Vera s/n, 46022, Valencia, Spain.

\begin{abstract}
This presentation details the results of the application of different methodologies (project based learning, oral presentations, case studies) for the measurement of two cross-disciplinary skills at the fourth course of the Bachelor's Degree in Business Administration and Management taught at the Escuela Politécnica de Alcoi of theUniversitat Politècnica de València.
\end{abstract}

Keywords: cross-disciplinary skills, measurement, higher education

\begin{abstract}
Resumen
En la presente ponencia se detallan los resultados obtenidos de la aplicación de diferentes metodologías (aprendizaje por proyectos, presentaciones orales, método del caso) para la medición de dos competencias transversales en una asignatura en el cuarto curso del grado de Administración y Dirección de Empresa impartida en la Escuela Politécnica de Alcoi de la Universitat Politècnica de València.
\end{abstract}

Palabras clave: competencias transversales, medición, enseñanza superior. 


\section{Introducción}

En los últimos años, la universidad está experimentando una serie de profundos cambios materializados en el proceso de convergencia hacia el Espacio Europeo de Enseñanza Superior (EEES), a través del cuál se busca un modelo más centrado en el alumno y en su aprendizaje.

En este sentido, es necesario dar respuesta a las nuevas necesidades que se plantean y que precisan de una respuesta en forma de la aplicación de nuevas metodologías didácticas y pedagógicas, que permitan una eficiente labor de aprendizaje por parte de los alumnos.

Por tanto, resulta fundamental que el profesorado disponga de las herramientas pedagógicas necesarias para poder facilitar el aprendizaje permanente del alumnado.

En este contexto, la incorporación de competencias transversales a la formación del alumnado adquiere una especial relevancia, siendo uno de los ejes fundamentales de la armonización universitaria en la Unión Europea (Casas, 2005).

De este modo, la elección y aplicación de las metodologías adecuadas, por parte del profesorado, se convierte en un elemento esencial, no sólo para lograr un aprendizaje efectivo por parte del alumno sino también para que adquiera y domine una serie de competencias tanto específicas de cada titulación como transversales.

En la presente ponencia, se presentan los resultados obtenidos de la aplicación de diferentes metodologías para la adquisición de tres competencias transversales en una asignatura del grado de Administración y Dirección de Empresas que se imparte en la Escuela Politécnica de Alcoi de la Universitat Politècnica de València (UPV).

\section{Antecedentes}

En los últimos años, el sistema universitario ha realizado una serie de importantes cambios como consecuencia de la incorporación del EEES. Entre ellos, destaca el paso de un sistema centrado en el profesor a un sistema mucho más centrado en el estudiante y en la consecución de los objetivos planteados en los estudios de las diferentes asignaturas.

Uno de estos cambios se materializa en la necesidad de adquirir una serie de competencias como resultado del aprendizaje.

Estas competencias, las podemos agrupar entre: aquellas que son específicas de un título académico o una profesión y aquellas que son más genéricas o transversales, las cuales se pueden definir como aquellas habilidades relacionadas con el desarrollo personal, que no dependen de un ámbito temático o disciplinario específico sino que aparecen en todos los dominios de la actuación profesional y académica (González y Wagenaar, 2003).

Es en estas últimas competencias son en las que vamos a centrar el presente trabajo, puesto que son habilidades necesarias e importantes para el desarrollo de cualquier profesión pero no se pueden encontrar de forma explícita en el desarrollo de asignaturas específicas.

En el caso de la Universitat Politècnica de València, se han definido 13 competencias transversales, a partir de las cuales se intentan cubrir los diferentes aspectos que recogen 
diferentes organismos como la agencia ABET, EUR-ACE, además de los reales decretos españoles.

En concreto, las competencias son: CT-1 comprensión e integración, CT2- Aplicación y pensamiento práctico, CT-3 Análisis y resolución de problemas, CT-4 Innovación, creatividad y emprendimiento, CT-5 Diseño y proyecto, CT-6 Trabajo en equipo y liderazgo, CT-7 Responsabilidad ética, medioambiental y profesional, CT-8 Comunicación efectiva, CT-9 Pensamiento crítico, CT-10 Conocimiento de problemas contemporáneos, CT-11 Aprendizaje permanente, CT-12 Planificación y gestión del tiempo y CT-13 Instrumental específica.

De estas 13 competencias, en el presente trabajo se analizarán los resultados obtenidos en la medición de 2 de ellas en las que la asignatura de Dirección Financiera del Grado de Administración de Empresas es un punto de control. En concreto se trata de:

- CT-01. Comprensión e integración.

- CT-05. Diseño y proyecto.

\subsection{La asignatura Dirección Financiera del grado en ADE}

La asignatura de Dirección Financiera se enmarca dentro del cuarto curso del grado en Administración y Dirección de empresas y se imparte en el campus de Alcoi de la Universitat Politècnica de València.

El objetivo principal del grado es formar a los alumnos para que sean capaces de gestionar, dirigir, asesorar y evaluar las organizaciones empresariales. Al mismo tiempo, también serán capaces de desarrollar su iniciativa emprendedora con el fin de llevar a cabo proyectos empresariales viables y sostenibles.

Los créditos establecidos para poder lograr la titulación son 240, de los cuales 60 corresponden a la formación básica, 127.5 obligatorios, 45 optativos y por último 7.5 al trabajo fin de grado.

La asignatura de Dirección Financiera es obligatoria, de 6 créditos y se imparte en el primer cuatrimestre del cuarto año de carrera.

En el año objeto del estudio, había 65 alumnos matriculados en la asignatura.

Al tratarse de una signatura perteneciente al último curso de la carrera, los alumnos que la cursan ya han cursado un número considerable de asignaturas, cada una de ellas con diferente profesorado, con diferentes metodologías de enseñanza y, por tanto, podemos considerar que los alumnos ya tienen un bagaje y una experiencia universitaria considerable.

La asignatura se estructura en 5 unidades didácticas, las cuales tienen un enfoque eminentemente práctico. Se trata de clases participativas en las que se realizan y analizan casos prácticos complementarios a la teoría así como de trabajos de búsqueda y análisis de información específica. 
Con respecto a la temática tratada en la asignatura, hay que tener en cuenta que las empresas crecen y se van expandiendo, se incrementa la competencia y, con ella, la presión por parte de los accionistas de aumentar los márgenes de beneficios. A esto se unen los cambios tecnológicos y la aparición de diferentes vías de inversión.

Mediante la asignatura de Dirección financiera se pretende dar a los alumnos las herramientas y los conocimientos necesarios para poder disponer de la estructura necesaria para la toma de decisiones financieras.

En ese sentido, los objetivos que se marcan son los siguientes:

- Conocer las bases teóricas para poder realizar una asignación eficiente de los recursos financieros de la empresa.

- Comprender la interrelación entre las decisiones de inversión y de financiación en el marco global de la empresa.

- Trasladar los conocimientos de la economía financiera a la realidad.

- Practicar el trabajo en grupo, elaboración de informes, etc.

Tabla 1. Datos descriptivos de la asignatura

\begin{tabular}{cccc}
\hline Asignatura & Titulación & Departamento & Alumnos \\
\hline Dirección financiera & $\begin{array}{c}\text { Grado en Administración } \\
\text { y Dirección de Empresas }\end{array}$ & $\begin{array}{c}\text { Economía y } \\
\text { Ciencias Sociales }\end{array}$ & 65 \\
\hline Centro & Créditos & Curso & Duración \\
\hline $\begin{array}{c}\text { Escuela Politécnica } \\
\text { Superior de Alcoi }\end{array}$ & 6 & $4^{\text {o }}$ & Cuatrimestral \\
\hline
\end{tabular}

Fuente: Elaboración propia

\section{Metodología}

Para la medición de la adquisición de las competencias transversales comentadas en los puntos anteriores se emplearon diferentes metodologías.

\subsection{Aprendizaje por proyectos}

En el aprendizaje por proyectos o PBL (Problem/Project Based Learning), los estudiantes consiguen los objetivos del curso a través de la realización de un trabajo de grupo que dura todo el período lectivo. A lo largo del desarrollo del trabajo, el profesor debe supervisar el trabajo del grupo, periódicamente, para controlar los avances así como ayudarles a resolver las dudas y problemas que les puedan surgir.

En esta metodología, el método de trabajo es más importante que el objeto del proyecto. El fin último es que los estudiantes sean capaces de analizar el problema a estudiar, proponer y aplicar la solución y evaluar los resultados. De esta forma, el producto final del proyecto se materializa en una producción tangible y en un informe escrito. 
El objetivo del trabajo propuesto en la asignatura de Dirección financiera es que los alumnos realicen un plan de viabilidad económico y financiero de una empresa y que, posteriormente, comenten los resultados del mismo a sus compañeros mediante una exposición oral.

La valoración del trabajo se realizará tanto de la memoria en la que se recoge toda la información del plan financiero así como de la presentación de la misma.

\subsection{Estudio de Casos}

Con la metodología del estudio de casos, a partir del análisis de casos reales se entrena a los alumnos en la práctica real. Cada grupo debe estudiar de forma analítica, y lo más exhaustivamente posible, un caso explicado en clase para poder extraer conclusiones.

Cada uno de los miembros puede aportar una solución diferente de acuerdo con sus conocimientos, experiencias y motivaciones. El caso puede narrarse, escribirse en hojas, presentarse en películas, etc. Para que el caso sea eficaz debe presentar elementos de incertidumbre, conflicto y urgencia para que despierte el interés su resolución. El tiempo de duración depende del caso de estudio.

Existen diferentes métodos para el estudio de casos: método de Harvard, caso dramatizado, caso simplificado, técnica de Pigors y redacción de casos por los alumnos (FernándezMarch, 2003).

En el caso de la asignatura de Dirección financiera, en el horario de aula, los alumnos analizan, sacan conclusiones y debaten sobre casos de estudio e informaciones aparecidas en prensa sobre los contenidos que se tratan en cada uno de los temas.

Como resultados de aprendizaje, el alumno comprende las causas y los efectos de las decisiones clave desde el punto de vista financiero, que se toman en las empresas. Además, el alumno es capaz de valorar las diferentes alternativas en materia de financiación e inversión en el entorno empresarial, siendo capaz de elegir la mejor de ellas.

Por último, el alumno a partir de los ejemplos y casos reales que se ven en clase es capaz de establecer relaciones entre los conceptos clave de la asignatura y la toma de decisiones.

\section{Resultados}

Para poder medir el nivel alcanzado en cada competencia transversal, la UPV ha definido los siguientes criterios:

- A: se supera con creces, de modo excelente.

- B: se alcanza satisfactoriamente, adquiriendo un nivel adecuado.

- C: está en proceso de adquisición, solo se ha alcanzado parcialmente.

- D: no se ha alcanzado, por el momento no se ha logrado el nivel mínimo. 
En el caso de la competencia CT 1, para alcanzar un nivel adecuado, el alumno debe describir, relacionar e interpretar situaciones y planteamientos sencillos.

Para valorar el nivel alcanzado por parte de los alumnos, se han evaluado los casos prácticos realizados en clase.

Para ello se ha tenido en cuenta:

- El grado de participación de los alumnos.

- Los resultados obtenidos en los casos.

- La interacción entre los alumnos y con el profesor.

- El aprovechamiento de los conocimientos adquiridos en la realización de cada caso práctico para poder plantear y desarrollar los siguientes casos prácticos.

Los resultados obtenidos han sido los siguientes:

Tabla 2. Comparación resultados competencia transversal 1

\begin{tabular}{ccccc}
\hline $\begin{array}{c}\text { Nivel } \\
\text { alcanzado }\end{array}$ & \multicolumn{2}{c}{ Alumnos } & \multicolumn{2}{c}{ Porcentaje } \\
\hline Curso 2016/17 & Curso 2017/18 & Curso 2016/17 & Curso 2017/18 \\
\hline D & 10 & 10 & $17 \%$ & $15 \%$ \\
C & 19 & 8 & $32 \%$ & $12 \%$ \\
B & 25 & 27 & $42 \%$ & $42 \%$ \\
A & 6 & 20 & $10 \%$ & $31 \%$ \\
\hline
\end{tabular}

Tal y como se puede apreciar en la tabla anterior, la aplicación de la metodología ha mejorado considerablemente el nivel alcanzado en la CT 1, frente a los resultados del curso anterior.

Con respecto a la competencia CT5, para poder lograr un nivel satisfactorio, los alumnos deben poder planificar proyectos de trabajo, en colaboración con otros alumnos, en situaciones poco estructuradas así como prever incidencias y riesgos.

Para la evaluación de esta competencia CT 5, se valora la realización de un trabajo en grupo. En el curso 2017/18 se hicieron 21 grupos, de los cuáles 19 estaban formados por 3 personas y 2 por 4 .

En la valoración del trabajo se han tenido en cuenta los siguientes ítems:

- Entrega del trabajo en la fecha establecida.

- Justificación del proyecto y objetivos.

- Desarrollo del proyecto e identificación de puntos de control. 
- Resultados y conclusiones.

- Presentación oral del trabajo ante el resto de la clase.

En la siguiente tabla, se pueden observar los resultados obtenidos en el curso 2017/18 y la comparación de éstos con respecto a los del curso anterior:

Tabla 3. Resultados competencia transversal 5

\begin{tabular}{ccccc}
\hline $\begin{array}{c}\text { Nivel } \\
\text { alcanzado }\end{array}$ & \multicolumn{2}{c}{ Alumnos } & \multicolumn{2}{c}{ Porcentaje } \\
\hline & Curso 2016/17 & Curso 2017/18 & Curso 2016/17 & Curso 2017/18 \\
\hline D & 8 & 10 & $13 \%$ & $15 \%$ \\
C & 14 & 12 & $23 \%$ & $18 \%$ \\
B & 26 & 16 & $43 \%$ & $25 \%$ \\
A & 12 & 27 & $20 \%$ & $42 \%$ \\
\hline
\end{tabular}

Tal y como se observa en la tabla anterior, se aprecia una mejoría notable, sobre todo en el porcentaje de alumnos que logran un mayor nivel de aprendizaje de la competencia, pasando de un $20 \%$ del curso $2016 / 17$ a un $42 \%$ en el curso siguiente.

\section{Conclusiones}

Como se ha comentado en los puntos anteriores, en los últimos años se han producido una serie de cambios en la enseñanza superior española a través de los cuáles se busca lograr un aprendizaje permanente por parte del alumnado. Estos cambios se han materializado, entre otros, en la necesidad de adquirir una serie de competencias como resultado del aprendizaje

Con el fin de lograr que los alumnos alcancen un nivel de aprendizaje adecuado de las diferentes competencias, es necesario emplear nuevas metodologías en las que se busque una mayor involucración por parte de los alumnos.

En el caso estudiado en la presente ponencia, la aplicación de metodologías activas ha permitido una mayor participación por parte del alumnado, consiguiendo de esta forma una mayor motivación del mismo y, al mismo tiempo, que un porcentaje muy elevado de los mismos alcancen los niveles deseados de las competencias analizadas.

\section{Referencias}

Casas, M. (2005). Nueva universidad ante la sociedad del conocimiento. Revista de Universidad y Sociedad del Conocimiento, 2 (2), pp. 1-18. 
Medición de competencias transversales en una asignatura de grado

Fernández March, A (2003). Formación pedagógica y desarrollo profesional de los profesores de universidad: Análisis de las diferentes estrategias. Revista de Educación, 331, pp. 171-197

González, J. y Wagenaar, R. (2003). Tuning educational structures in Europe. Informe final Proyecto Piloto, Fase Uno. Bilbao: Universidad de Deusto.

Hernanz, M.L, et al. (2004). Marc general per a la integració europea. Barcelona: Agència per a la Qualitat del Sistema Universitari a Catalunya.

Universitat Politècnica de València (2019). Competencias transversales. Recuperado de http://www.upv.es/contenidos/COMPTRAN/ 